\title{
The InterMine Android app: Cross-organism genomic data in
}

\section{your pocket [version 1; peer review: 1 approved, 1 approved with reservations]}

\author{
Daria Komkova(iD), Rachel Lyne (iD), Julie Sullivan, Yo Yehudi (iD), Gos Micklem (ii)
}

Department of Genetics, University of Cambridge, Downing Street, Cambridge, CB2 3EH, UK

V1 First published: 22 Nov 2018, 7:1837

https://doi.org/10.12688/f1000research.17005.1

Latest published: 07 Jun 2019, 7:1837

https://doi.org/10.12688/f1000research.17005.2

\section{Abstract}

InterMine is a data integration and analysis software system that has been used to create both inter-connected and stand-alone biological databases for the analysis of large and complex biological data sets. Together, the InterMine databases provide access to extensive data across multiple organisms. To provide more convenient access to these data from Android mobile devices, we have developed the InterMine app, an application that can be run on any Android mobile phone or tablet. The InterMine app provides a single interface for data access, search and exploration of the InterMine databases. It can be used to retrieve information on genes and gene lists, and their relatives across species. Simple searches can be used to access a range of data about a specific gene, while links to the InterMine databases provide access to more detailed report pages and gene list analysis tools. The InterMine app thus facilitates rapid exploration of genes across multiple organisms and kinds of data.

Keywords

Android app, Genomics data, Gene search, InterMine

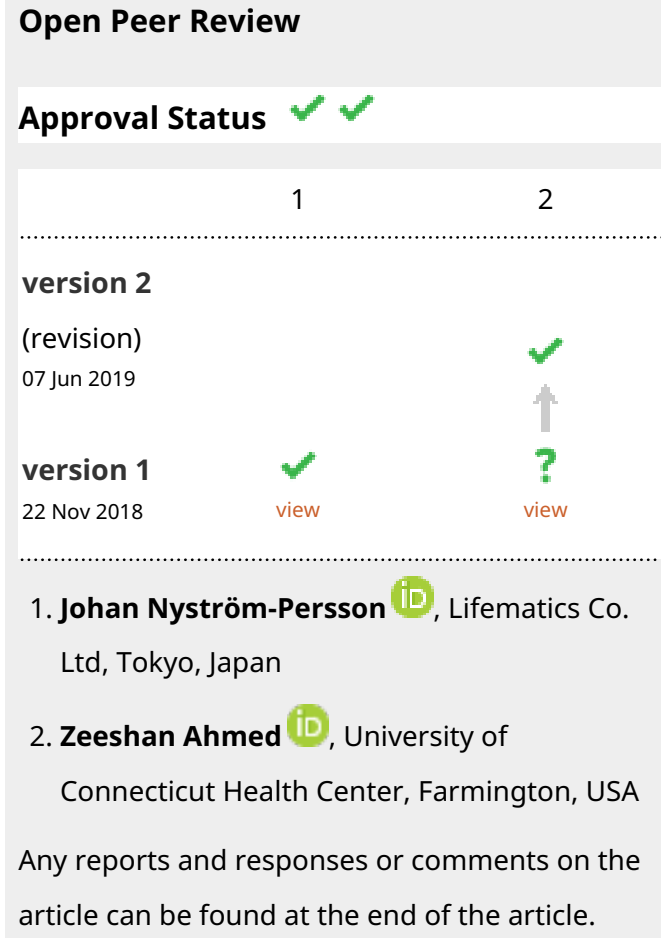

Corresponding author: Gos Micklem (gm263@cam.ac.uk)

Author roles: Komkova D: Software; Lyne R: Writing - Original Draft Preparation; Sullivan J: Supervision, Writing - Review \& Editing; Yehudi Y: Software; Micklem G: Funding Acquisition, Supervision, Writing - Review \& Editing

Competing interests: No competing interests were disclosed.

Grant information: This work was supported by the Wellcome Trust (Grant number: 099133).

The funders had no role in study design, data collection and analysis, decision to publish, or preparation of the manuscript.

Copyright: @ 2018 Komkova D et al. This is an open access article distributed under the terms of the Creative Commons Attribution License, which permits unrestricted use, distribution, and reproduction in any medium, provided the original work is properly cited.

How to cite this article: Komkova D, Lyne R, Sullivan J et al. The InterMine Android app: Cross-organism genomic data in your pocket [version 1; peer review: 1 approved, 1 approved with reservations] F1000Research 2018, 7:1837

https://doi.org/10.12688/f1000research.17005.1

First published: 22 Nov 2018, 7:1837 https://doi.org/10.12688/f1000research.17005.1 


\section{Introduction}

InterMine is a data integration and analysis software system that has been used to create a suite of both inter-connected and stand-alone biological databases for the analysis of large and complex biological data sets. InterMine databases have been developed for the major model organisms budding yeast ${ }^{1}$, nematode worm, fruit $\mathrm{fly}^{2}$, zebrafish, mouse ${ }^{3}$ and $\mathrm{rat}^{4}$, (which we will refer to as the Model Organism Database (MOD-) InterMines, together with a human database and databases for plants, bees and wasps $^{5}$, cows ${ }^{6}$, Medicago truncatula ${ }^{7}$, mitochondrial proteomics ${ }^{8}$ and drug targets $^{9}$ (Table 1; https://registry.intermine.org/). Together, the InterMine databases provide access to extensive data across multiple organisms (for full listings of data included see the website for each individual InterMine, Table 1). To provide more convenient access to these data from Android mobile devices, we have developed the InterMine app ${ }^{10}$, an application that can be run on any Android mobile phone or tablet.

The InterMine app provides a single interface for data access, search and exploration of the above databases. It can be used to retrieve information on genes and gene lists, and their relatives across species. Simple searches can be used to access a range of data about a specific gene, while links to the InterMine databases provide access to more detailed report pages and gene list analysis tools.
The app is available from the Google Play Store at https://play. google.com/store/apps/details?id=org.intermine.app.

\section{Methods}

\section{Data sources}

The InterMine app allows users to search a default subset of InterMine data warehouses (Table 1). This list is configurable, and so users are able to refine or add mines to match their interests. See https://registry.intermine.org for the full list of known public InterMine instances.

InterMine databases typically integrate data from many resources. For instance BioGRID ${ }^{11}$, IntAct ${ }^{12}$, UniProt ${ }^{13}$, and can include high quality curated data (from the Model Organism Databases), genome-wide high-throughput data and data from smaller more focused studies (See individual InterMine websites for more details)

Search and analysis

The InterMine app provides several ways to search and explore the data available, including a keyword search, sets of pre-defined template searches and list analysis functionality. These features are described in more detail in the use case section.

Table 1. InterMine databases available through the InterMine app by default. The list can be configured in the app.

\begin{tabular}{|c|c|c|c|}
\hline Database & Data & Organisation & url \\
\hline FlyMine & Fruit fly & InterMine & http://www.flymine.org \\
\hline HumanMine & Human & InterMine & http://www.humanmine.org \\
\hline MedicMine & $\begin{array}{l}\text { Medicago } \\
\text { truncatula }\end{array}$ & JCVI & http://medicmine.jcvi.org \\
\hline MouseMine & MGI & MGI & http://www.mousemine.org \\
\hline PhytoMine & $\begin{array}{l}\text { Multiple plant } \\
\text { genomes }\end{array}$ & Phytozome & https://phytozome.jgi.doe.gov \\
\hline RatMine & Rat & RGD & http://ratmine.mcw.edu \\
\hline TargetMine & $\begin{array}{l}\text { Support } \\
\text { for drug } \\
\text { discovery }\end{array}$ & $\mathrm{NIBIO}$ & http://targetmine.mizuguchilab.org \\
\hline ThaleMine & Thale cress & Araport & https://apps.araport.org/thalemine \\
\hline WormMine & Nematode & WormBase & http://intermine.wormbase.org/tools/wormmine \\
\hline YeastMine & $\begin{array}{l}\text { Budding } \\
\text { yeast }\end{array}$ & SGD & http://yeastmine.yeastgenome.org/yeastmine \\
\hline ZebrafishMine & Zebrafish & ZFIN & http://zebrafishmine.org/ \\
\hline HymenopteraMine & $\begin{array}{l}\text { Various } \\
\text { Hymenoptera }\end{array}$ & $\begin{array}{l}\text { University of } \\
\text { Missouri }\end{array}$ & http://hymenopteragenome.org/hymenopteramine \\
\hline BovineMine & Bos taurus & $\begin{array}{l}\text { Bovine Genome } \\
\text { Database Project }\end{array}$ & http://bovinegenome.org/bovinemine \\
\hline
\end{tabular}




\section{MyMine accounts}

InterMine databases allow users to create an account through which they can, between sessions, store lists and searches. The InterMine App therefore allows users to log in to any accounts they hold on the underlying databases, so enabling user-created lists to be accessed.

\section{Favourite genes}

Users are also able to mark genes in search results as favourite. These genes are stored on the Android device and can be accessed without needing to $\log$ in to any of the underlying databases.

\section{Implementation}

\section{Communication}

The InterMine App draws all data from the RESTful Application Programming Interfaces (APIs) that InterMine databases provide $^{14}$. RoboSpice, an Open Source (Apache 2.0 Licence) Android communications library ${ }^{15}$, provides core network communication functionality. Data are loaded asynchronously over HTTP or HTTPS, depending on the preferred protocol of the database being accessed. For performance enhancement, most web service responses are stored on the device if headers state an appropriate cache lifetime.

When the app receives a JSON response from the web service, it transforms the data from a table-structured format to a more hierarchical view, which presents data more effectively on smaller-screened mobile devices.

\section{Authentication}

Each InterMine database is discrete and often maintained by different organisations. If a user wishes to authenticate with multiple InterMines - perhaps to view private gene lists stored on different databases - they will need to provide separate authentication details for each InterMine database. However this is only necessary once, as after a user has successfully authenticated in a given InterMine via a username/password pair, the app retrieves and stores an API authentication token. This ensures that the user can authenticate in the future without having to re-enter or store sensitive password details.

All of the user configuration settings and authentication tokens are stored locally on the device via SharedPreferences, Android's dedicated settings storage mechanism ${ }^{16}$.

\section{Internal storage}

Tabular data, such as favourite genes within the app, are not suited to the key/value pair storage used in SharedPreferences ${ }^{17}$, and therefore are stored within an SQLite database on the user's device. Data stored include the InterMine instance the data originated from, the (e.g.) gene's identifiers, description, organism, and genomic coordinates.

\section{Search}

Keyword search is available across lists, templates and gene search results. Search results from different databases are presented to the user as a single result set, sorted by the search relevance score generated by each originating database. Search results can be shared via email, instant messaging, and other sharing media in text format, using Android's ACTION_SEND Intent functionality ${ }^{18}$.

\section{Advanced information}

InterMine also includes advanced analysis tools - particularly data visualisations - which may not be available via the API. To access the extended information about genes or gene lists, a user can load InterMine's advanced report pages within the app itself. This is implemented via Android's WebView ${ }^{19}$ functionality which allows live web pages to be embedded in an application (for example, Figure 2 shows an example of an embedded InterMine WebView).

\section{Operation}

The app is implemented in accordance with Google Material Design $^{20}$ guidelines, providing a predictable environment for the user, and also supports Android version 4.0 and above, ensuring it is able to run on over $99 \%$ of active Android phones as of November $2018^{21}$.

\section{Use case}

Cross-organism gene search

The keyword search simultaneously searches all InterMine databases selected through the settings option. Thus, a crossorganism overview of data available for further investigation is provided. Link-outs from the search results to each originating InterMine database provide access to detailed gene report pages. These pages collate information integrated for that gene and typically include functional summaries, ontology annotation, pathway, expression, interaction and disease data and links to additional related data.

As an example, searching for 'dopamine' returns dopaminerelated genes from PhytoMine, MouseMine, HumanMine, TargetMine, FlyMine, RatMine, ZebrafishMine, WormMine, YeastMine, ThaleMine and HymenoperaMine (Figure 1; see Table 1 for urls). Selecting a gene from the results, for instance the human gene DRD4 (dopamine receptor D4) displays summary information about the gene, with a link to the full gene report available from the HumanMine database. Here we learn, for example, that polymorphisms in the DRD4 gene are associated with the disorder attention deficit hyperactivity disorder (ADHD) (Figure 2), a condition associated with low dopamine levels. The search results therefore facilitate rapid exploration across multiple organisms and kinds of data. 


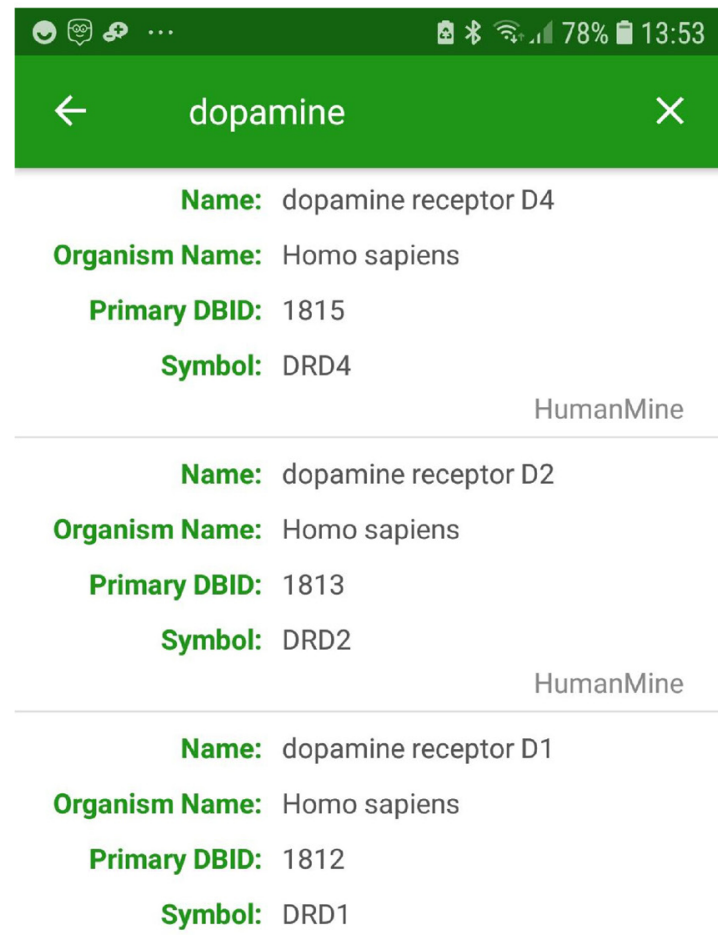

HumanMine
Organism Name: Drosophila melanogaster
Primary DBID: FBgn0034136
Symbol: DAT
Name: dopamine beta-hydroxylase

Figure 1. Example keyword search results. A search for dopamine returns genes from PhytoMine, MouseMine, HumanMine, FlyMine, TargetMine, RatMine, ZebrafishMine, YeastMine and ThaleMine. A section of the results from HumanMine and FlyMine is shown.

\section{Template searches}

In addition to cross-organism gene search, the InterMine databases provide libraries of pre-built searches, called template searches. Such searches provide a user-friendly interface where the parameters for search filters can be specified. These templates range from simple searches, such as for a specified gene (or genes), return the corresponding Gene Ontology terms (represented as "Gene $\rightarrow$ Gene Ontology terms"), to more complex searches combining data of more than one type, such as "Tissue + interaction $\rightarrow$ genes", which returns any genes expressed in the specified tissue that also interact with the product of a specified gene. Templates from each InterMine database are available within the InterMine App. The results are provided with a simple keyword search to facilitate further data browsing. For instance, continuing the above dopamine example, we can use a template search to identify genes in Drosophila associated with ADHD: on the templates page for the FlyMine database, we find the template "Disease $\rightarrow$ Human genes and
Orthologues" (Figure 3). This template allows one to specify disease names that contains "attention deficit", and on running the template, this returns the disease Attention deficit-hyperactivity disorder along with associated human genes and their orthologues from the available InterMine databases. Using the ability to search within the results we are able to verify that the human gene we are interested in (DRD4) is associated with this condition, and that this gene has a predicted orthologue in Drosophila melanogaster, FBgn0053517, Dop2R. Through such iterative searching we can continue our investigation of this fly orthologue to identify, for instance, interacting partners, pathway and Gene Ontology annotations.

\section{Lists}

InterMine databases are especially suited to the analysis of lists of genes or other entities. Users can create their own lists, which can be stored between sessions if the user has an account for the relevant InterMine database. Again, direct links from 
A

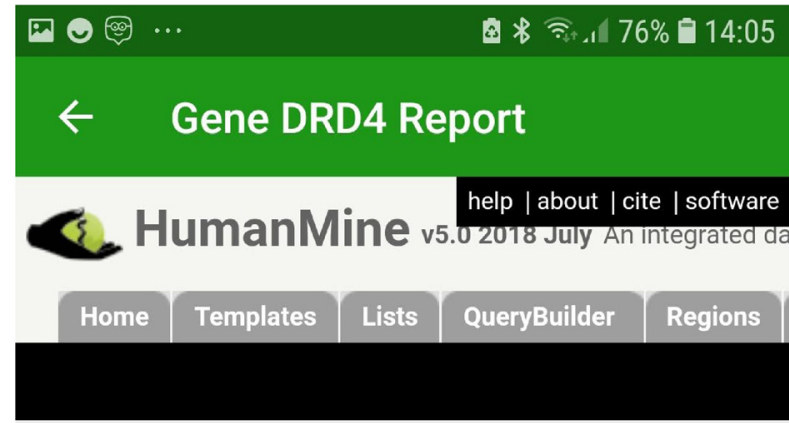

Gene : DRD4 Homo sapiens

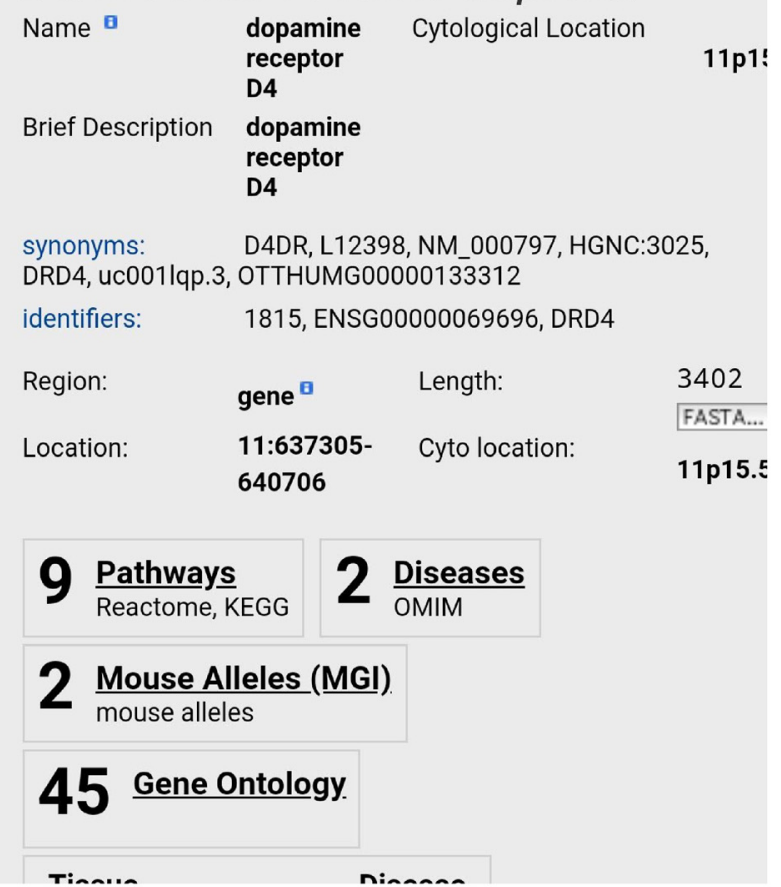

B

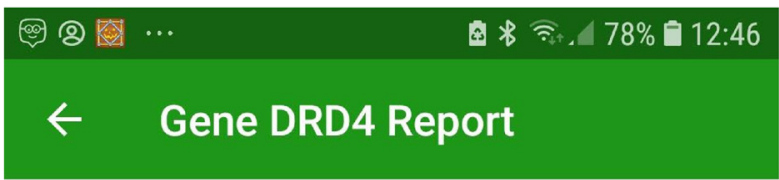

\section{Disease}

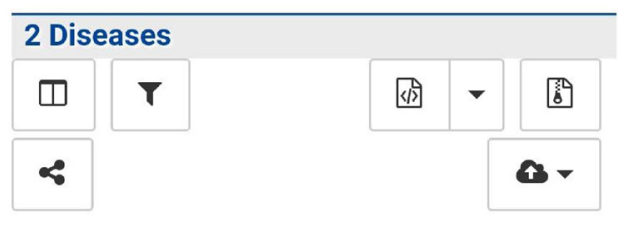

1 to 2 of 2

\footnotetext{
Gene (Hum OR Rat) --> Mouse Allele

(Phenotype) (11 rows)
}

Mouse Gene --> Allele [Phenotype] (0 rows)

\section{Homology}

Figure 2. The human DRD4 report page from the HumanMine webapp, displayed in the InterMine app. (a) This view shows the first part of the report page with a summary of the Gene identifiers and data available. (b) This view shows the Disease section of the report page.

lists to the underlying InterMine databases provide access to list analysis tools, for instance enrichment statistics that help identify surprising properties, such as publications that cite an unexpectedly large number of the list members, or GO terms or protein domains that are associated with an unexpectedly large number of list members.

Public lists, which are typically interesting sets of genes derived from publications and other studies, are often provided by the database operators. For instance, in FlyMine, eleven of the public lists provide sets of genes whose expression increases at defined times during drosophila embryogenesis, as derived from Hooper $e t a l^{22}$. Further lists show genes that are expressed at increased levels in various adult fly tissues according to data from the FlyAtlas resource ${ }^{23}$. Within one of these sets, PL FlyAtlas_brain_top, we can identify a set of genes upregulated in the brain. Checking within this list, we find that the dopamine receptor gene Dop2R (FBgn0053517) identified above is present. By following the link to the corresponding list analysis page on the underlying FlyMine website, and examining the enrichment statistics, we find that the Dop $2 R$ gene is part of a set in which the Gene Ontology term dopamine receptor signaling pathway (GO:0007212) occurs unexpectedly frequently (p-value 0.001303 , with Holm-Bonferroni correction). It is also apparent that two other fly dopamine receptors, DoplRl (FBgn0011582) and DoplR2 (FBgn0266137) are also found in this list (Figure 5). 


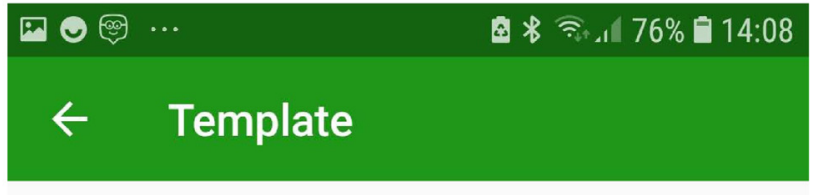

Disease --> Human Gene and Orthologues

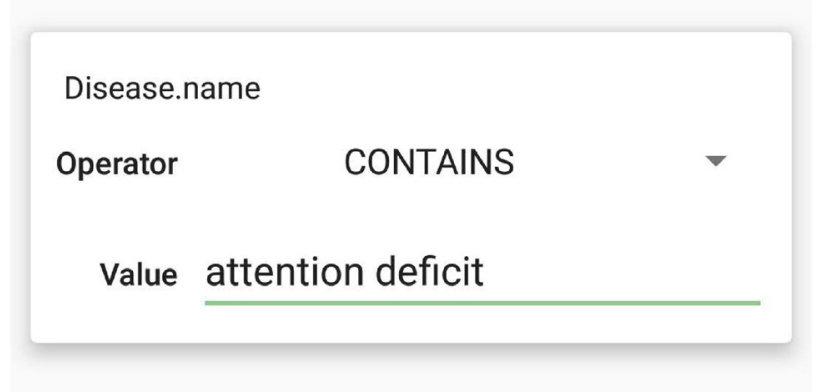

\section{SHOW RESULTS}

Figure 3. A FlyMine template search, "Disease -> Human genes and Orthologues". The disease name is an editable constraint, in this case set to search for human genes associated with diseases with a name that "contains" Attention deficit, as well as their orthologues.

\section{Conclusions}

The InterMine app provides a convenient way of searching for biological information across many model organism and other databases, allowing an overview of gene function and gene relationships to be pursued. Importantly, the InterMine app reduces the effort required to obtain data available in a range of InterMine databases by removing the need to visit each one individually. Further development of the app is planned, including

\begin{tabular}{|c|c|}
\hline 国今 & $\Delta \diamond \curvearrowright$ \\
\hline \multicolumn{2}{|c|}{ Disease --> Human... } \\
\hline \multicolumn{2}{|r|}{ Disease } \\
\hline \multicolumn{2}{|l|}{ Identifier } \\
\hline \multirow[t]{4}{*}{ Name } & ATTENTION \\
\hline & DEFICIT-HYPERACTIVITY \\
\hline & DISORDER \\
\hline & Genes \\
\hline \multicolumn{2}{|l|}{ Symbol } \\
\hline \multicolumn{2}{|c|}{ Name dopamine receptor D4 } \\
\hline \multicolumn{2}{|c|}{ Homologues } \\
\hline \multirow{2}{*}{\multicolumn{2}{|c|}{$\begin{array}{l}\text { Homologue. FBgn0053517 } \\
\text { Primary Identifier }\end{array}$}} \\
\hline & \\
\hline \multirow{2}{*}{\multicolumn{2}{|c|}{$\begin{array}{c}\text { Type orthologue } \\
\text { Data Sets }\end{array}$}} \\
\hline & \\
\hline \multirow[t]{2}{*}{ Name } & TreeFam data set \\
\hline & Homologue \\
\hline Organism Name & Drosophila melanogaster \\
\hline
\end{tabular}

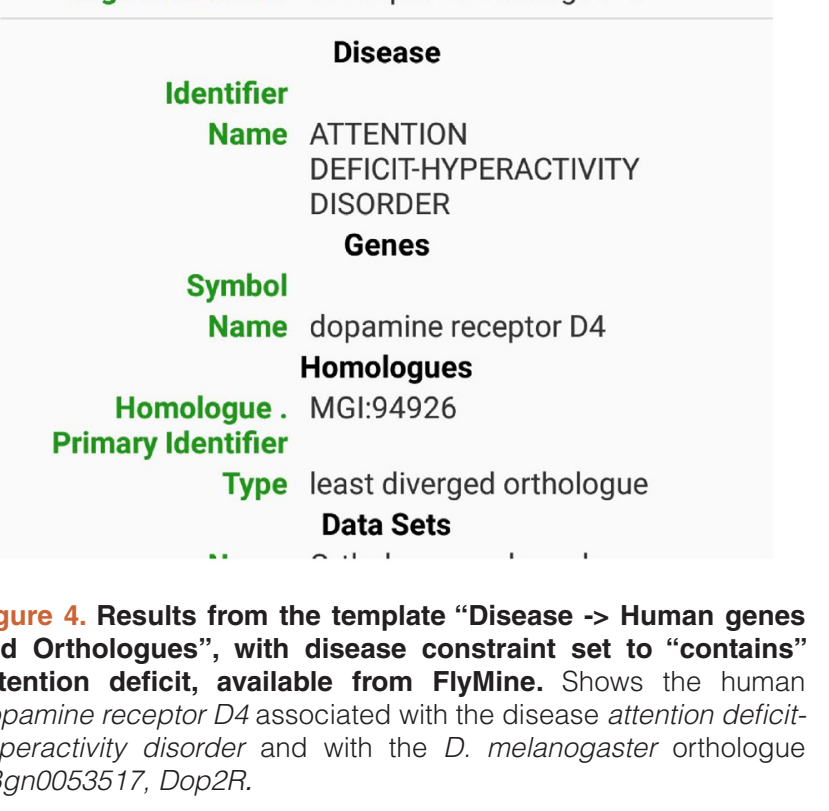

a single sign-in for all of the InterMine instances through oAuth2; further search and analysis capabilities including extending the keyword search to include all data types (instead of just genes); better cross-InterMine search result ordering; an offline mode with data cached in a local database for access when no internet connection is available, and a more sophisticated query construction capability for more advanced users. 


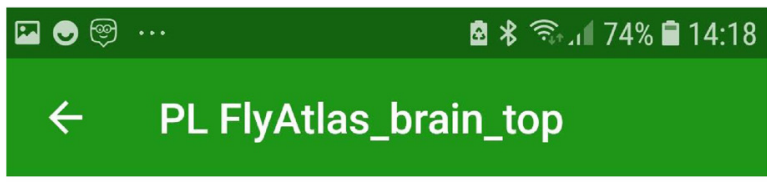

\section{Gene Ontology Enrichment}

GO terms enriched for items in this list.

Number of Genes in this list not analysed in this widget: 10

\begin{tabular}{|c|c|c|c|c|c|}
\hline \multicolumn{3}{|c|}{ Test Correction } & \multicolumn{2}{|c|}{ Max p-value } & Ontolog \\
\hline \multicolumn{3}{|c|}{ Holm-Bonferroni } & 0.05 & $\boldsymbol{v}$ & biolog \\
\hline \multicolumn{3}{|c|}{ Background population } & \multicolumn{2}{|c|}{ Normalise by length $\boldsymbol{i}$} & \\
\hline Default & Change & & & Normalise & \\
\hline View & Download & & & & \\
\hline$\square$ & O Term & & & & $\mathrm{p}-\mathrm{V}$ \\
\hline
\end{tabular}

\section{dopamine receptor signalin!} pathway

3 Genes:

FBgn0011582 FBgn0053517 FBgn026613

View results Create list

Figure 5. Gene Ontology enrichment analysis of the FlyMine public list PL FlyAtlas_brain_top. A set of three genes (FBgn0011582 (Dpo1R1), FBgn0053517 (dop2R) and FBgn0266137 (Dop1R2) are enriched for the GO term dopamine receptor signaling pathway.

\section{Data availability}

All data underlying the results are available as part of the article and no additional source data are required.

\section{Software availability}

The InterMine app is available from the Google Play Store: https://play.google.com/store/apps/details?id=org.intermine. app.

Source code available from: https://github.com/intermine/intermineandroid.
Archived source code at time of publication: https://doi. org/10.5281/zenodo. $1478646^{10}$.

License: GNU General Public License v2.

Grant information

This work was supported by the Wellcome Trust (Grant number: 099133).

The funders had no role in study design, data collection and analysis, decision to publish, or preparation of the manuscript. 
1. Balakrishnan R, Park J, Karra K, et al:: YeastMine--an integrated data warehous for Saccharomyces cerevisiae data as a multipurpose tool-kit. Database (Oxford). 2012; 2012: bar062.

PubMed Abstract | Publisher Full Text | Free Full Text

2. Lyne R, Smith R, Rutherford K, et al:: FlyMine: an integrated database for Drosophila and Anopheles genomics. Genome Biol. 2007; 8(7): R129. PubMed Abstract | Publisher Full Text | Free Full Text

3. Motenko H, Neuhauser SB, O'Keefe M, et al:: MouseMine: a new data warehouse for MGI. Mamm Genome. 2015; 26(7-8): 325-330. PubMed Abstract | Publisher Full Text | Free Full Text

4. Wang SJ, Laulederkind SJ, Hayman GT, et al.: Analysis of disease-associated objects at the Rat Genome Database. Database (Oxford). 2013; 2013: bat046. PubMed Abstract | Publisher Full Text | Free Full Text

5. Elsik CG, Tayal A, Diesh CM, et al.: Hymenoptera Genome Database: integrating genome annotations in HymenopteraMine. Nucleic Acids Res. 2016; 44(D1): D793-800.

PubMed Abstract | Publisher Full Text | Free Full Text

6. Elsik CG, Unni DR, Diesh CM, et al:: Bovine Genome Database: new tools for gleaning function from the Bos taurus genome. Nucleic Acids Res. 2016; 44(D1): D834-9.

PubMed Abstract | Publisher Full Text | Free Full Text

7. Krishnakumar V, Kim M, Rosen BD, et al.: MTGD: The Medicago truncatula genome database. Plant Cell Physiol. 2015; 56(1): e1. PubMed Abstract | Publisher Full Text

8. Smith AC, Robinson AJ: MitoMiner v3.1, an update on the mitochondria proteomics database. Nucleic Acids Res. 2016; 44(D1): D1258-61. PubMed Abstract | Publisher Full Text | Free Full Text

9. Chen YA, Tripathi LP, Mizuguchi K: TargetMine, an integrated data warehouse for candidate gene prioritisation and target discovery. PLoS One. 2011; 6(3): e17844.

PubMed Abstract | Publisher Full Text | Free Full Text

10. Komkova D, Yehudi Y, Lyne R, et al:: Intermine-android App (Version v1.0.10) Zenodo. 2018.

http://www.doi.org/10.5281/zenodo.1478646

11. Chatr-Aryamontri A, Breitkreutz BJ, Oughtred R, et al.: The BioGRID interaction database: 2015 update. Nucleic Acids Res. 2015; 43(Database issue): D470-8. PubMed Abstract | Publisher Full Text | Free Full Text
12. Kerrien $\mathrm{S}$, Aranda $\mathrm{B}$, Breuza $\mathrm{L}$, et al.: The IntAct molecular interaction database in 2012. Nucleic Acids Res. 2012; 40(Database issue): D841-6.

PubMed Abstract | Publisher Full Text | Free Full Text

13. UniProt Consortium: UniProt: a hub for protein information. Nucleic Acids Res. 2015; 43(Database issue): D204-12.

PubMed Abstract | Publisher Full Text | Free Full Text

14. Kalderimis A, Lyne R, Butano D, et al.: InterMine: extensive web services for modern biology. Nucleic Acids Res. 2014; 42(Web Server issue): W468-72. PubMed Abstract | Publisher Full Text | Free Full Text

15. stephanenicolas: stephanenicolas/robospice. GitHub. (Accessed: 10th November 2016). Reference Source

16. Saving Key-Value Sets |Android Developers. (Accessed: 10th November 2016). Reference Source

17. Saving Data in SQL Databases |Android Developers. (Accessed: 10th November 2016). Reference Source

18. Sending Simple Data to Other Apps |Android Developers. (Accessed: 10th November 2016). Reference Source

19. Building Web Apps in WebView | Android Developers. (Accessed: 10th November 2016) Reference Source

20. Material Design for Android | Android Developers. (Accessed: 10th November 2016).

Reference Source

21. Dashboards | Android Developers. (Accessed: 9th November 2016). Reference Source

22. Hooper SD, Boué S, Krause R, et al:: Identification of tightly regulated groups of genes during Drosophila melanogaster embryogenesis. Mol Syst Biol. 2007; 3 :

PubMed Abstract | Publisher Full Text | Free Full Text

23. Robinson SW, Herzyk P, Dow JA, et al:: FlyAtlas: database of gene expression in the tissues of Drosophila melanogaster. Nucleic Acids Res. 2013; 41(Database issue): D744-50.

PubMed Abstract | Publisher Full Text | Free Full Text 


\section{Open Peer Review}

\section{Current Peer Review Status:}

\section{Version 1}

Reviewer Report 18 February 2019

https://doi.org/10.5256/f1000research.18593.r43497

(C) 2019 Ahmed Z. This is an open access peer review report distributed under the terms of the Creative Commons Attribution License, which permits unrestricted use, distribution, and reproduction in any medium, provided the original work is properly cited.

\section{Zeeshan Ahmed}

University of Connecticut Health Center, Farmington, CT, USA

Authors have produced a smart phone app, and I would like to congratulate them for good work.

Here, I provide some comments:

Introduction is brief; there is no background and rational provided, which can justify the need of such application. Moreover, there are no details provided of similar smart phone applications and other related platforms (e.g. web, desktop etc.). I would suggest to address these points comprehensively and add comparative analysis of their app with other related applications, including a table based on common and variable features would be helpful.

As authors are interested in publishing their app as a scientific contribution, it's important to have scientific justifications and discussion. At this time paper is more like a brief report.

Methodology; why Android based smart phone app, why not iOS?

Is data behind the app (collection of databases) freely accessible to the users, so they can download, and even verify the results and with other referenced databases? If not, then mention it in the paper, and give reasons for that. As this is an open source work, and data is collected from multiple open sources, its expected to have access to the data linked at the backend.

Authors have mentioned list of databases, it's important to mention licensing information of those database, to avoid any conflict of interest. Moreover, its important to clearly mention it in the conflict of interest section.

Author's contribution are missing, it's also important to list those.

I would suggest to write supplementary material and there explain the app in detail (step-by-step). Guide a new user as to how to get access to the app and how each interface can be used, and what are expected inputs/outputs. Furthermore, if word count restriction does not allow, then 
further extend supplementary material and provide comprehensive details of software implementation, database design and data workflow with rational for choosing those options. Make some diagrams (based on software engineering concepts) explaining design and implementation parts.

I would suggest authors to also mention:

1. Current limitations of the app.

2. Current advantages of the app, which signifies it technically and scientifically.

3. What were the major technical, non-technical, and scientific difficulties they have faced while designing and developing this app.

4. Future recommendations, in their view and for other readers.

Regarding Figures; Figure 1 seems isolated. I would rather suggest make one good multi panel Figure, and add 1, 2, 3, 4 to that.

Is the rationale for developing the new software tool clearly explained?

No

Is the description of the software tool technically sound?

Partly

Are sufficient details of the code, methods and analysis (if applicable) provided to allow replication of the software development and its use by others?

Partly

Is sufficient information provided to allow interpretation of the expected output datasets and any results generated using the tool?

Partly

Are the conclusions about the tool and its performance adequately supported by the findings presented in the article?

Partly

Competing Interests: No competing interests were disclosed.

Reviewer Expertise: Bioinformatics

I confirm that I have read this submission and believe that I have an appropriate level of expertise to confirm that it is of an acceptable scientific standard, however I have significant reservations, as outlined above.

Author Response 22 May 2019

Rachel Lyne, University of Cambridge, Downing Street, UK

Authors have produced a smart phone app, and I would like to congratulate them for good work. 
We thank the reviewer for his detailed comments on the manuscript. We have made several changes to the manuscript and have made further comments below which we hope address the points made:

Here, I provide some comments:

Introduction is brief; there is no background and rational provided, which can justify the need of such application. Moreover, there are no details provided of similar smart phone applications and other related platforms (e.g. web, desktop etc.). I would suggest to address these points comprehensively and add comparative analysis of their app with other related applications, including a table based on common and variable features would be helpful.

As authors are interested in publishing their app as a scientific contribution, it's important to have scientific justifications and discussion. At this time paper is more like a brief report.

As the development of mobile apps for biology is still in its infancy and as this provided a new and novel way of accessing the data in InterMine, it's development was largely exploratory as we did not know at the outset how much demand for the app there would be. The very popular iOS app developed by the Saccharomyces Genome database (utilizing their yeastMine webservices) suggested that this could become a popular way in which data could be accessed (Wong et al; PMID: 23396302). Indeed, the InterMine app has already had well over one hundred downloads and we hope that with continued development this number will increase. We have added a paragraph to the introduction to cover these points.

Methodology; why Android based smart phone app, why not iOS?

An iOS app has been developed independently and will be published separately as the technology is quite different.

Is data behind the app (collection of databases) freely accessible to the users, so they can download, and even verify the results and with other referenced databases? If not, then mention it in the paper, and give reasons for that. As this is an open source work, and data is collected from multiple open sources, its expected to have access to the data linked at the backend.

Search results from within the app can be shared as described under "Search" in the Implementation section. Further export options are available if the full InterMine database is accessed via the app.

We have added a sentence to under "search" of the Implementation section to make this clearer to the reader.

Authors have mentioned list of databases, it's important to mention licensing information of those database, to avoid any conflict of interest. Moreover, its important to clearly mention it in the conflict of interest section.

All the InterMine databases are open source and provide open access to the data within 
them.

However there are some restrictions for commercial use of some of the data and each individual InterMine should be consulted for its policy. We have added a sentence to the methods section to make this clearer to the reader.

\section{Author's contribution are missing, it's also important to list those.}

Author contributions were provided in the original paper and are available under the author details.

I would suggest to write supplementary material and there explain the app in detail (step-by-step). Guide a new user as to how to get access to the app and how each interface can be used, and what are expected inputs/outputs. Furthermore, if word count restriction does not allow, then further extend supplementary material and provide comprehensive details of software implementation, database design and data workflow with rational for choosing those options. Make some diagrams (based on software engineering concepts) explaining design and implementation parts.

We feel that the three key aspects of the interface have been fully explained through the use-case and accompanying screenshots. Further details for all aspects of the InterMine interface can be found in previous publications. The InterMine database design and the webservices used to power the InterMine app have also been previously described. The following sentences have been added to the manuscript to direct readers more readily to this information:

Introduction (to use-case)

The following use case introduces each of the three key features of the InterMine app; Gene search, Templates and Lists with an example of their use. Further details of these InterMine features have been fully described in previous publications from several InterMine databases $(1,2,3,5,6,8,9)$.

Added as first sentence under Communication:

The InterMine database design and the webservices used to power the InterMine app have been previously described $(1,14)$.

Competing Interests: No competing interests were disclosed.

Reviewer Report 10 January 2019

https://doi.org/10.5256/f1000research.18593.r42072

(c) 2019 Nyström-Persson J. This is an open access peer review report distributed under the terms of the Creative Commons Attribution License, which permits unrestricted use, distribution, and reproduction in any medium, provided the original work is properly cited. 


\section{Johan Nyström-Persson}

Lifematics Co. Ltd, Tokyo, Japan

The authors describe the InterMine Android app, which makes data available from InterMine applications on Android devices. Significantly, it is able to combine data from multiple data warehouses, and users can add their own InterMine instances to this list.

The source code is GPL licensed and available on GitHub, and the source code as it was at the time of publication has also been archived.

For this review, unfortunately I did not have access to an Android device, so I assume that the application functions as described.

The app seems to contain a carefully chosen set of core features to enable quick searches, for example by gene or template, and presents combined search results in a streamlined user interface. It is also possible to access gene lists. The WebView feature to display visualisations should also be useful, although most likely the user experience here is harder to control by nature (owing to the different widgets that may be available in InterMine instances being accessed).

I am curious as to how the app would behave if one queries different InterMine instances that have very different response times and displays the combined results. Hopefully, the app would display results incrementally as they are available, instead of waiting for the slowest instance to reply and then combining the results.

The authors have also taken reasonable care to handle authentication details and transmitted information securely.

In summary, the InterMine Android App should be useful for querying InterMine instances on the go from handheld devices, but also for combining data from different instances in a way that, as far as I know, has not been possible until now. I recommend acceptance of this report.

Is the rationale for developing the new software tool clearly explained?

Yes

Is the description of the software tool technically sound?

Yes

Are sufficient details of the code, methods and analysis (if applicable) provided to allow replication of the software development and its use by others?

Yes

Is sufficient information provided to allow interpretation of the expected output datasets and any results generated using the tool?

Yes

Are the conclusions about the tool and its performance adequately supported by the findings presented in the article? 
Yes

Competing Interests: No competing interests were disclosed.

Reviewer Expertise: Bioinformatics, software development, genomics

I confirm that I have read this submission and believe that I have an appropriate level of expertise to confirm that it is of an acceptable scientific standard.

The benefits of publishing with F1000Research:

- Your article is published within days, with no editorial bias

- You can publish traditional articles, null/negative results, case reports, data notes and more

- The peer review process is transparent and collaborative

- Your article is indexed in PubMed after passing peer review

- Dedicated customer support at every stage

For pre-submission enquiries, contact research@f1000.com 